\title{
Lack of Cardiac Differentiation in c-kit-Enriched Porcine Bone Marrow and Spleen Hematopoietic Cell Cultures Using 5-Azacytidine
}

\author{
M.L. Ramirez ${ }^{a}$ I.M. McMorrow ${ }^{a}$ T.M. Sanderson ${ }^{a, b}$ C.J. Lancos ${ }^{a}$ \\ Y.-L. Tseng ${ }^{a}$ D.K.C. Cooper ${ }^{a}$ F.J.M.F. Dor ${ }^{a, c}$ \\ ${ }^{a}$ Transplantation Biology Research Center, Massachusetts General Hospital/Harvard Medical School, \\ Boston, Mass., ' Immerge BioTherapeutics, Cambridge, Mass., USA; ' ${ }^{\mathrm{b}}$ Department of Surgery, \\ Erasmus Medical Center, Rotterdam, The Netherlands
}

\section{Key Words}

5-Azacytidine $\cdot$ Bone marrow $\cdot \mathrm{c}$-kit $\cdot$ Hematopoietic stem cells $\cdot$ Spleen $\cdot$ Pig

\begin{abstract}
The adult spleen is a source of early hematopoietic stem cells (HSC). We therefore studied whether culturing spleen or bone marrow (BM) HSC in medium containing 5-azacytidine could induce a cardiac phenotype. c-kit enrichment and depletion of adult pig spleen and BM mononuclear cells were obtained by magnetic bead separation using biotinylated pig stem cell factor (c-kit ligand). Cells were incubated with 5-azacytidine for $24 \mathrm{~h}$ and refreshed with 5-azacytidine-free medium every $48 \mathrm{~h}$. Western blot was used to detect cardiac troponin and myosin heavy chains. Although 5-azacytidine treatment led to the formation of ball-like cell clusters in both c-kit-enriched populations, these clusters showed no rhythmic contractions (beating), as observed by others. Furthermore, neither cardiac troponin nor myosin was detected in cells derived from either source. Our methodology and treatment with 5-azacytidine did not induce cardiac gene expression in porcine HSC derived from either pig spleen or BM.
\end{abstract}

\section{Introduction}

Although the presence of hematopoietic stem cells (HSC) is well characterized in the bone marrow (BM), we have recently shown that the spleens of pigs, baboons and humans are also sources of early HSC [Dor et al., 2004]. Although CD34 is often used as a marker of the earliest HSC, it is also known that, in the sequence of events associated with hematopoietic differentiation, porcine cells expressing c-kit (c-kit+) contain pluripotent HSC as well [Le Guern et al., 2003]. Through the use of the cobblestone area-forming cell (CAFC) assay, an assay that defines the presence of early HSC [Ploemacher et al., 1989; Breems et al., 1994], we document-

\begin{tabular}{ll} 
Abbreviations used in this paper \\
\hline BM & Bone marrow \\
CAFC & Cobblestone area-forming cell \\
HBSS & Hanks balanced salt solution \\
HSC & Hematopoietic stem cell \\
MACS & Magnetic cell separation \\
MSC & Bone marrow stromal cell \\
SCF & Stem cell factor
\end{tabular}


ed an equal frequency of these earliest stem cells in BM and spleen [Dor et al., 2004]. The question was then raised whether these c-kit+ stem cells might reflect an early pluripotent state that could be directed away from hematopoietic fate.

Recent work at understanding the repair of infarcted myocardial tissue and the ability of fetal myocardial cells to potentially repair cardiac tissue has generated interest in this field as a potential therapeutic option [Leor et al., 1996]. At the same time, ethical ramifications and technical problems of fetal tissue acquisition have generated great interest in adult stem cell populations and the ability of these cells to dedifferentiate and be directed toward other fates [Friedenstein et al., 1987; Ashton et al., 1980; Bennett et al., 1991; Rickard et al., 1994; Ferrari et al., 1998]. Along these lines, the ability of 5-azacytidine, a cytosine analog, to induce altered gene expression and in vitro cellular rhythmic contractions, associated with a cardiac phenotype, has been well documented [Constantinides et al., 1977; Constantinides et al., 1978; Taylor and Jones, 1979; Fukuda, 2001]. Fukuda [2001] has demonstrated that regenerative cardiomyocytes can be generated in the rat from BM stromal cells (MSC) using 5-azacytidine, and Tomita et al. [2002] have demonstrated that porcine heart function improved following the infusion of 5-azacytidine-treated MSC into infarcted myocardium.

In view of the work of Rangappa et al. [2003], which demonstrated the ability of adipose-derived rat mesenchymal stem cells to differentiate towards cardiac fate, a similar approach was applied to the c-kit-expressing HSC from the spleen and BM. The spleen, like the BM, is a hematopoietic tissue of mesodermal origin and, as such, may serve as a repository for stem cells that retain their plasticity. Recent work by Kodama et al. [2003], which showed that injected mouse splenocytes contribute to the regeneration of functional pancreatic islet cells, suggests that the transdifferentiation of splenic cells to an alternate phenotype may, in fact, be possible.

Based on the shared embryonic origins of the splenic and BM HSC, and further supported by their similar CAFC frequencies at 12 weeks of culture [Dor et al., 2004], we attempted transformation of c-kit-expressing HSC from spleen and BM cells toward cardiac fate by an approach that has been reported to be successful by others working with mesenchymal stem cells.

\section{Materials and Methods}

\section{Animals}

Four naive pigs were obtained from the Massachusetts General Hospital herd of partially inbred major histocompatibility complex-defined miniature swine (aged 3-5 months), whose characteristics have been described in detail previously [Sachs, 1992]. All procedures were in accordance with the Principles of Laboratory Animal Care formulated by the National Society for Medical Research and the Guide for the Care and Use of Laboratory Animals produced by the Institute of Laboratory Animal Resources and published by the National Institutes of Health (NIH publication No. 86-23, revised 1996).

\section{Primary Culture of Porcine c-kit Cells}

Spleen and sternal BM samples were obtained in a sterile fashion from adult pigs immediately following the administration of an overdose of pentobarbital to induce euthanasia. The samples were washed in Hanks balanced salt solution (HBSS; Gibco, Grand Island, N.Y., USA) and minced in a dish of RPMI 1640 culture medium (Mediatech, Herndon, Va., USA). The resulting suspension was passed through a 40-micron filter (BD Biosciences, San Jose, Calif., USA), diluted 1:1 with HBSS, and mononuclear cells isolated using a gradient of lymphocyte separation medium (ICN Biomedicals, Aurora, Ohio, USA). The buffy coats were harvested and residual erythrocytes lysed using ACK lysing buffer (Cambrex Bio Science, Walkersville, Md., USA). The cells were resuspended in culture medium consisting of RPMI 1640, 15\% fetal bovine serum, penicillin $(1 \mathrm{U} / \mathrm{ml})$, streptomycin $(1 \mathrm{mg} / \mathrm{ml})$, gentamicin $(0.05 \mathrm{mg} /$ $\mathrm{ml})$, amphotericin B $(0.34 \mu \mathrm{g} / \mathrm{ml})$ (all from Gibco), Hepes buffer (10 $\mathrm{m} M$; Mediatech) and glutamine (2 $\mathrm{m} M$; Gibco). The cells were incubated with 1:40 porcine biotinylated recombinant porcine stem cell factor (c-kit ligand) (BioTransplant, Charlestown, Mass., USA) [Le Guern et al., 2003], then with magnetic cell separation (MACS) anti-biotin magnetic microbeads (MiltenyiBiotec, Auburn, Calif., USA), and separated over MACS LS columns (MiltenyiBiotec) with the negative fraction being retained for use in the c-kit-depleted (c-kit-) cultures. The c-kit+ cells were then eluted for use as HSC. Cells $\left(1 \times 10^{6}\right)$ were removed from each population for fluorescence-activated cell sorting analysis to evaluate purification of the different populations. Both the c-kit+ and c-kit- cell populations from the spleen and BM were counted and separately seeded onto $25-\mathrm{cm}^{2}$ flasks at a concentration of $1 \times 10^{6}$ cells $/ \mathrm{ml}$ in the same culture medium as above. At $24 \mathrm{~h}$, the cells were trypsinized $(0.25 \%$ trypsin with EDTA; Mediatech) and resuspended in the above culture medium.

\section{Treatment with 5-Azacytidine}

Half of the cells from both c-kit+ and c-kit- cultures were transferred from the culture flasks into 6-well plates in culture medium (as described above) with the addition of 5-azacytidine (Sigma-Aldrich, St. Louis, Mo., USA) at a concentration of $9 \mu M$. This was the same concentration used in previous experiments in which a cardiac phenotype was observed. The other half was cultured in the same manner, in the absence of 5-azacytidine. After $24 \mathrm{~h}$ incubation with 5 -azacytidine, $40 \%$ of the medium was removed from each well and replaced with fresh culture medium without 5-azacytidine. The medium (40\%) was again removed and replaced after 36 and $48 \mathrm{~h}$ in order to titrate down the concentration of 5-azacytidine as quickly as possible. Culture medium was thereafter 
changed every $48 \mathrm{~h}$. The cells were observed under an inverted light microscope at several time points over 5 weeks.

Phenotypic Assessment of Cell Cultures by Flow Cytometry

Cell suspensions for flow cytometry from the BM and spleen were resuspended in flow cytometry buffer, consisting of HBSS, $0.1 \%$ BSA and $0.4 \%$ sodium azide (diluted $1: 1: 1$ ). Pig immunoglobulin $\left(5 \mu \mathrm{g} / 10^{6}\right.$ cells) was added to prevent nonspecific binding. Cells were labeled with biotinylated recombinant porcine stem cell factor; biotinylated 12.2.2. (IgM) and fluorescein isothiocyanate 36.7.5 (IgG2a) were used as negative isotype-matched controls.

\section{Western Blot and Visualization}

After 5 weeks in culture, cells were removed from the bottom of the plates using $0.25 \%$ trypsin with EDTA and lysed at a concentration of $100 \mu 1 / 10^{6}$ cells using a solubilization buffer, consisting of $100 \mathrm{~m} M \mathrm{NaCl}, 1.0 \%$ SDS, $50 \mathrm{~m} M$ tetraethylammonium chloride (pH 7.4), $1 \mathrm{~m} M$ DTT, $1 \mathrm{~m} M$ PMSF and Protease Inhibitor Cocktail for use with mammalian cell and tissue extracts 1:1,000 (SigmaAldrich). The resulting lysates were loaded under reducing conditions onto SDS-PAGE gels (7.5 and $12.5 \%$ acrylamide for myosin and troponin staining, respectively). The proteins were then transferred to nitrocellulose membrane (Hybond C, Amersham Biosciences, Piscataway, N.J., USA) and stained with Ponceau Red to assess protein transfer. The nitrocellulose was incubated in blocking buffer (PBS with 10\% fetal bovine serum and 0.1\% Tween) overnight, washed and incubated for $1 \mathrm{~h}$ with either mouse anti-myosin heavy chain (with known pig reactivity, $0.2 \mu \mathrm{g} / \mathrm{ml}$ ) (Biodesign International, Saco, Me., USA) or anti-troponin I (with known pig reactivity, $0.2 \mu \mathrm{g} / \mathrm{ml}$ ) (Novus Biologicals, Littleton, Colo., USA) monoclonal antibodies diluted in the same manner. The membranes were washed in blocking buffer and incubated with horseradish peroxidase-conjugated anti-mouse IgG (1:25,000; Southern Biotechnology Associates, Birmingham, Ala., USA). The SuperSignal West Pico chemiluminscent substrate kit (Pierce Biotechnology, Rockford, Ill., USA) was used to develop the blots.

\section{Results}

\section{c-kit Purity by MACS Cell Separation}

The isolation of c-kit+ populations from the BM was substantially more efficient than that from the spleen tissue (table 1). Following cell separation, in the BM, there was a significant difference in mean c-kit expression between the populations: $66.01 \%$ ( $\pm 14.67 \mathrm{SD}$ ) c-kit+ cells in the enriched population and $3.31 \%( \pm 1.76 \mathrm{SD})$ in the depleted population $(p=0.01)$. In the spleen, the separation came close to, but did not achieve, statistical significance; enriched cultures contained a mean of 19.98\% $( \pm 7.07 \mathrm{SD}) \mathrm{c}-\mathrm{kit}+$ cells, and depleted samples contained $3.44 \%$ ( $\pm 1.06 \mathrm{SD}) \mathrm{c}-\mathrm{kit}+$ cells $(\mathrm{p}=0.05)$. When BM and spleen were compared, there was no statistical difference between the respective c-kit-enriched populations $(\mathrm{p}=$ $0.07)$ or the respective $\mathrm{c}-$-kit-depleted populations $(\mathrm{p}=$ $0.93)$.
Table 1. Enrichment of c-kit HSC in porcine BM and spleen

\begin{tabular}{lccc}
\hline Tissue & \multicolumn{2}{l}{ c-kit, $\%$} & \\
\cline { 2 - 4 } & unseparated & enriched & depleted \\
\hline BM & & & \\
Trial 1 & & & \\
Trial 2 & 20.38 & 81.84 & 5.34 \\
Trial 3 & 7.36 & 52.88 & 2.34 \\
Mean & 8.06 & 63.31 & 2.28 \\
SD & 11.93 & 66.01 & 3.32 \\
Spleen & 7.32 & 14.67 & 1.75 \\
Trial 1 & & & \\
Trial 2 & 4.99 & 12.1 & 2.77 \\
Trial 3 & 4.49 & 25.79 & 2.89 \\
Mean & 8.79 & 22.04 & 4.67 \\
SD & 6.09 & 19.98 & 3.44 \\
& 2.35 & 7.07 & 1.06 \\
\hline
\end{tabular}

c-kit unseparated $=\mathrm{c}-\mathrm{kit}+$ cells prior to separation over column; c-kit enriched $=\mathrm{c}$-kit + cells in enriched cultures; $\mathrm{c}$-kit-depleted $=$ c-kit+ cells in depleted cultures.

${ }^{1}$ Samples that showed multinucleate ball masses.

\section{Growth and Morphology of Cultures prior to}

\section{5-Azacytidine Treatment}

Following separation, there was a gross difference in the overall morphology of the cells from the two tissues. BM c-kit+ isolates had cells that were larger and well rounded compared with splenic c-kit+ isolates, where the cells were much smaller and had more variation in shape (fig. 1). In the c-kit-cultures, the BM cells were somewhat smaller than the corresponding c-kit+ BM cells, but still remained well rounded. Morphologically, splenic c-kitcultures were not as distinct from splenic c-kit+ cells as in the BM. Both splenic populations were of the same relative size and featured variation in cell morphology that included both smaller, well-rounded cells and other more spindle-shaped cells.

\section{Effects of 5-Azacytidine Treatment}

\section{Bone Marrow}

Regardless of exposure to 5-azacytidine, there were substantial morphological variations between the samples (table 2). In only one of the three 5-azacytidine-exposed, c-kit-enriched BM samples were multinucleated fusion bodies visualized, resembling those observed by Taylor and Jones [1979] or Rangappa et al. [2003]. In this single culture, at 1 week, there were several separate foci of fibroblast or triangle-like morphology. This growth continued through week 2 , with the joining of separate 

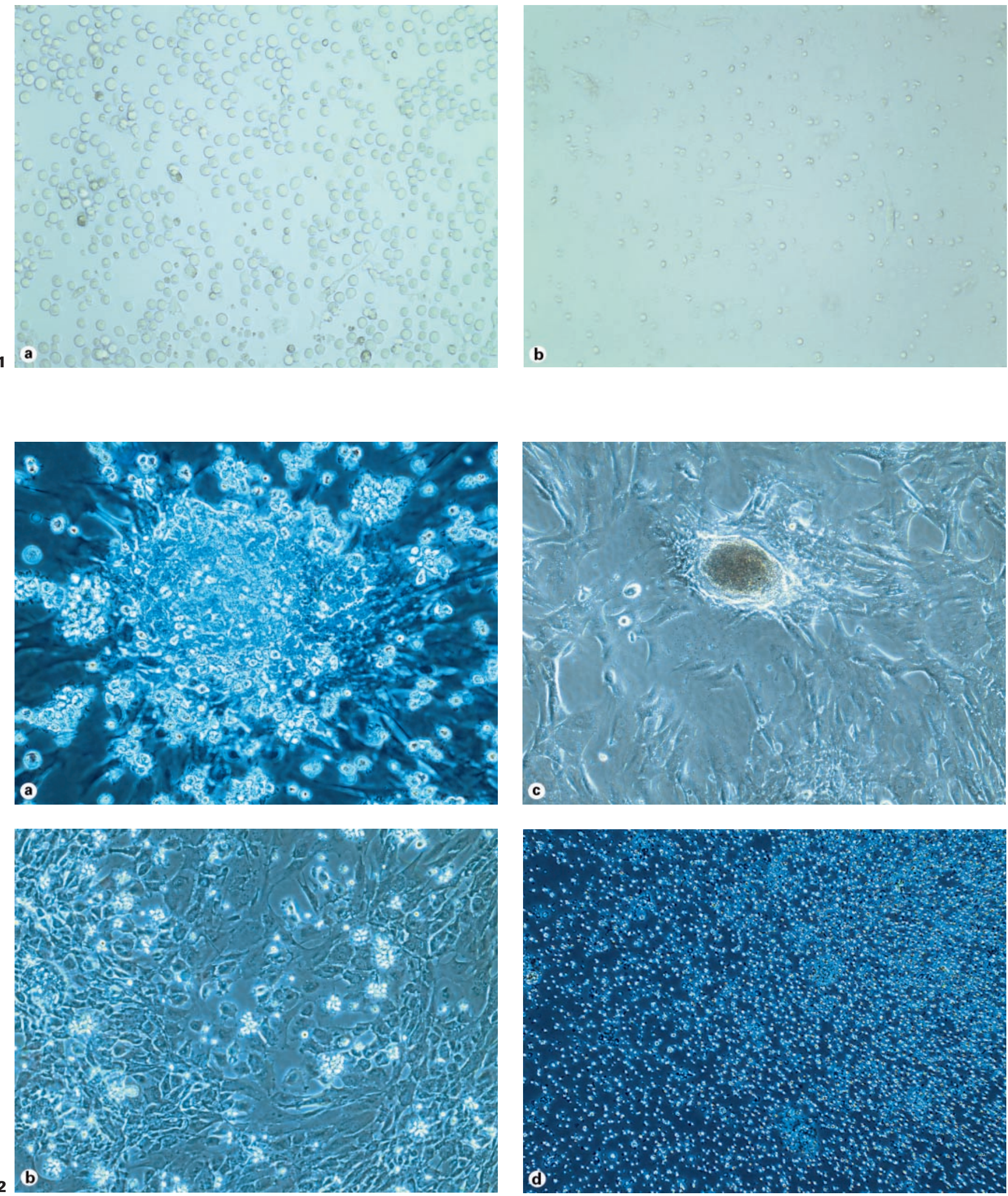
Table 2. Relationship between exposure to 5-azacytidine and morphology

\begin{tabular}{llll}
\hline $\begin{array}{l}\text { Source } \\
\text { of cells }\end{array}$ & c-kit & $\begin{array}{l}\text { 5-Aza- } \\
\text { cytidine }\end{array}$ & $\begin{array}{l}\text { Morphology of cells } \\
\text { (3 samples in each group) }\end{array}$ \\
\hline BM & enriched & + & $\begin{array}{l}\text { 1 with fusion bodies } \\
\text { 2 with fibroblastic activity but no fusion bodies }\end{array}$ \\
& depleted & + & 3 with fibroblastic activity but no fusion bodies \\
& enriched & - & 3 with comparatively decreased fibroblastic activity but no fusion bodies \\
& depleted & - & 3 with comparatively decreased fibroblastic activity but no fusion bodies \\
\hline Spleen & enriched & + & 1 with fusion bodies \\
& & & 1 with fibroblastic activity and no fusion bodies \\
& & & 1 stagnant with no growth \\
& depleted & + & 3 stagnant with no growth \\
& enriched & - & 3 stagnant with no growth \\
depleted & - & 1 with strong fibroblastic-like growth but no fusion bodies \\
& & & 2 stagnant with no growth \\
\hline
\end{tabular}

fibroblastic foci and the presence of multinucleated masses by day 23 (fig. 2a). The masses continued to grow in size until day 29 , when they assumed a constant size without further change in morphology. These masses ranged from 1 to 9 per well and were only present in foci with high fibroblastic activity. At no point during the 5 weeks of culture was either rhythmic or nonrhythmic beating observed in any of the structures.

The other two 5-azacytidine-exposed, c-kit-enriched BM samples showed similar fibroblastic activity through week 2, but there was no subsequent evidence of multinucleated masses. A growth pattern without multinucleated masses was consistently observed in each of the c-kit-depleted cultures exposed to 5-azacytidine in addition to both groups of cultures that were not exposed to 5 -azacytidine at any time (fig. 2b). Growth in cultures not exposed to 5-azacytidine was relatively decreased, with some cultures showing foci of growth that did not join with neighboring colonies.

Fig. 1. BM and spleen c-kit-enriched cultures. One day after separation over a magnetic column, BM c-kit-enriched cultures show larger and more rounded cells compared with spleen c-kit-enriched cultures. a $14 \times$; b $14 \times$.

Fig. 2. BM and spleen c-kit-enriched populations showed various morphologies at day 21 following exposure to 5-azacytidine. a BM culture showing a multinucleate fusion body. b BM culture showing fibroblastic growth, but no fusion bodies. c Spleen culture showing a multinucleate fusion body similar to that in $\mathbf{a}$. d Spleen culture showing stagnation, with no growth or fibroblastic activity. a $7 \times$; b $14 \times ;$ c $14 \times ;$ d $14 \times$.

Lack of Differentiation Using 5-Azacytidine

\section{Spleen}

In the spleen, there was considerably greater variation (table 2). Multinucleated cell masses similar to those seen in the BM were visualized in only one culture that was enriched for c-kit and exposed to 5-azacytidine. The observed changes followed a time course similar to that in the corresponding BM culture (fig. 2c). However, of the other two c-kit-enriched samples exposed to 5-azacytidine, fibroblastic activity was present in only one. The other culture remained static with cells fixed to the bottom of the well, but with no visible sign of cellular extensions (fig. $2 \mathrm{~d})$. This same growth was observed in the cultures that were exposed to 5-azacytidine and depleted of c-kit, as well as in each of the cultures that were neither exposed nor enriched. In the c-kit-depleted populations that were not exposed to 5-azacytidine, strong fibroblastic activity was present in only one sample, which displayed the same clustered cell masses as in the c-kit-enriched population.

\section{Western Blot Analysis}

Analysis by SDS-PAGE did not detect the presence of either cardiac troponin I or cardiac myosin heavy chain in any of the cultures generated, regardless of exposure to 5-azacytidine. In those single BM and spleen cultures that showed multinucleated cell masses, expression of these proteins was again negative. Control samples of freshly processed porcine cardiac tissue were used as positive controls for the myosin heavy chain and troponin antibodies; fresh splenic tissue was used as a negative control in each gel (fig. 3). Extended exposures of each blot were done in order to detect low 


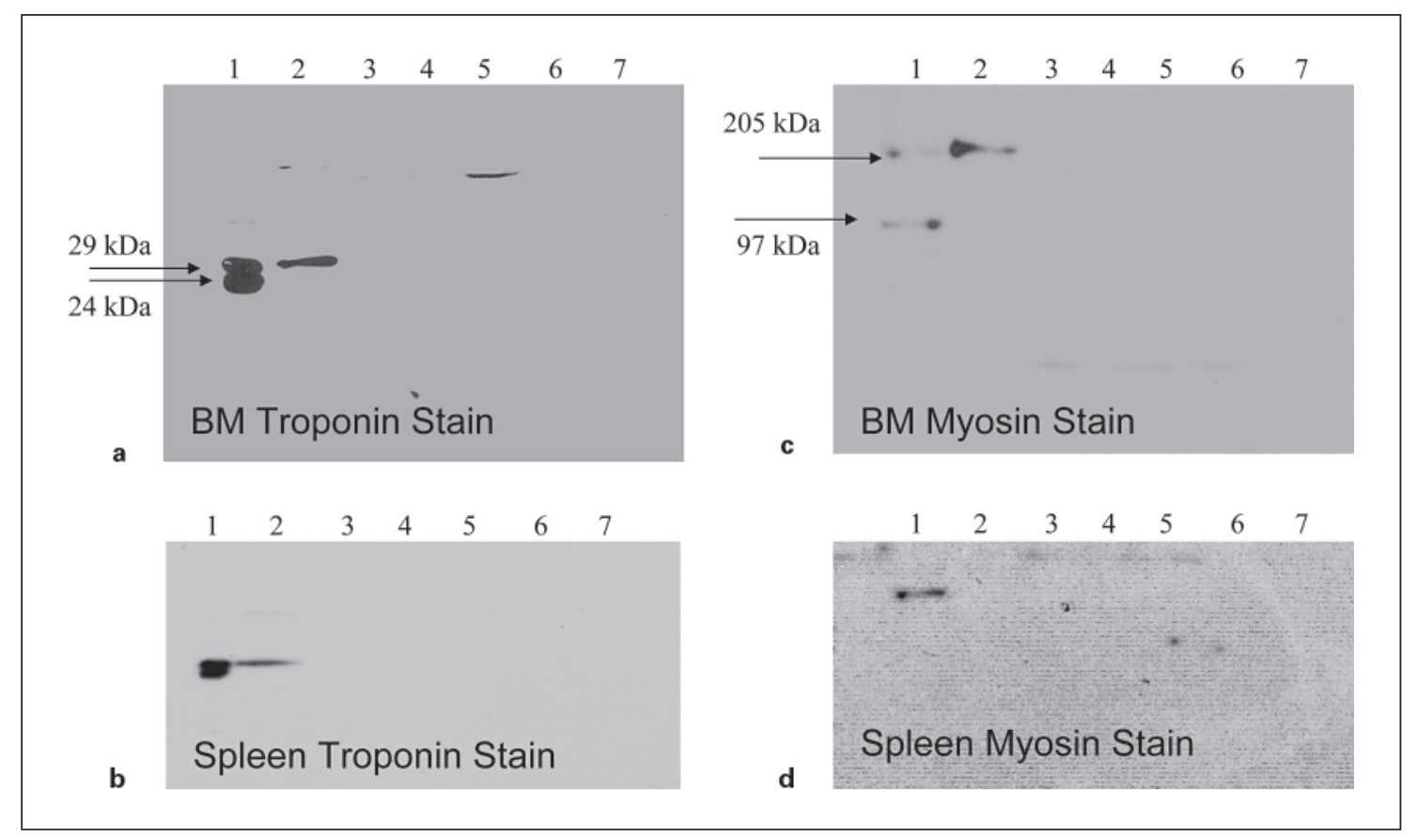

Fig. 3. Absence of expression of troponin I or myosin in BM or spleen cultures on Western blot. BM and spleen stained for troponin $(\mathbf{a}, \mathbf{b})$; BM and spleen stained for myosin $(\mathbf{c}, \mathbf{d}) .1$ = Molecular marker; 2 = cardiomyocytes (control); 3 = BM c-kit enriched + 5-azacytidine, 4 = BM c-kit depleted + 5-azacytidine; 5 = spleen c-kit enriched, no 5-azacytidine; 6 = spleen c-kit depleted, no 5-azacytidine; 7 = homogenized spleen (control). Lanes 2 and 7 are positive and negative control, respectively.

level expression of either myosin or troponin, but no signals were observed in any of the samples (data not shown).

\section{Discussion}

The ability of 5-azacytidine to induce novel gene expression has been documented by several different groups over the last few decades [Constantinides et al., 1977; Constantinides et al., 1978; Taylor and Jones, 1979; Fukuda, 2001; Tomita et al., 2002; Rangappa et al., 2003]. Although Constantinides et al. [1977] were the first to describe the appearance of multinucleated striated muscle cells in non-myeloblast precursors following 5-azacytidine treatment, it was Makino et al. [1999] who first described the appearance of cardiomyocytes from immortalized MSC. Because it was necessary for cell lines to be immortalized for this conversion, any clinical utility of this methodology remained questionable. However, soon after this, Tomita et al. [1999] reported that MSC in a primary culture were also capable of achieving this phenotype, and
Rangappa et al. [2003] have recently reported that mesenchymal stem cells derived from fatty tissue in rats assume a rhythmic beat consistent with cardiac phenotype following exposure to 5-azacytidine. However, there is some conflict in the literature, as Liu et al. [2003] have reported an inability to reproduce these results in rat primary MSC cultures. Their work suggested that, unless the cell line was immortalized, a cardiac phenotype was not achievable. The present study was an attempt to add further experience by assessing the utility of 5-azacytidine to influence the fate of porcine pluripotent HSC.

Based on our previous work, it was known that a small fraction of c-kit+ cells that were also CD9-, and therefore nonmobilized, were present in the spleen [Dor et al., 2004]. The work of Le Guern et al. [2003], which showed that c-kit was a marker for HSC and that this cell population displays early engraftment capacity, lead us to believe that these cells might be very early stem cells present in the spleen. Indeed, CAFC assays indicated that the earliest stem cells in the spleen were present at an equal frequency to those in the BM, raising the question of whether this population, or another splenic cell popula- 
tion, may have the same potential as the mesenchymal stem cells and MSC described above. It was a central premise to our study whether enrichment for c-kit would improve the conversion of HSC to cardiac phenotype, if this were at all achievable. Although CD34 is a widely used marker for HSC in rodents and humans, there is not yet a commercially available anti-CD34 antibody for pigs. However, c-kit+ expression is known to have a strong correlation with stem cell activity and can therefore be reliably used as a stem cell marker in pigs [Le Guern et al., 2003; Dor et al., 2004]. By using both BM and spleen as sources of HSC, it has been possible to compare the results, not only between these two sources, but also between splenic HSC and the MSC described by others [Makino et al., 1999; Tomita et al., 1999; Liu et al., 2003; Rangappa et al., 2003]. If such an increase in efficacy had been seen, there would have been some argument for including c-kit as a possible marker for increased plasticity and potential conversion to cardiomyocytes. If c-kit expression were unrelated to phenotype conversion, there would have been a similar frequency of cardiac conversion regardless of the enrichment status, provided that the cells were still exposed to 5-azacytidine. If this were the case, it was hypothesized that separation over a magnetic column would not affect the results. However, it should be noted that we were unable to achieve the same efficacy in separation in the spleen as in the BM, despite the same methodology. This may result from the fact that, although spleen cells express c-kit, they do not express c-kit ${ }^{\text {high }}$ as do the HSC of the BM [Dor et al., 2004]. This distinction may be one cause for the difference in purity of the cells obtained from the MACS columns. Although the effect of this variation remains uncertain, we believed that it was more important to maintain an identical, standardized separation protocol for both tissues. Thus, we included any cells expressing c-kit, whether c-kit ${ }^{\text {high }}$ or c-kit ${ }^{\text {low }}$, in the c-kit+ group, and only those cells failing to express any c-kit at all in the c-kit- group.

Our results were consistent with those observed by Liu et al. [2003]. Although we observed a fibroblastic growth pattern similar to Rangappa et al. [2003], our cultured cells not only failed to beat, they also failed to express cardiac-specific troponin and myosin proteins at any level. The gross appearance of the multinucleate structures in one BM and one spleen from separate animals was most consistent with the formation of chondrocyte structures first described by Taylor and Jones [1979]. However, as noted by Chacko et al. [1969] and Benya et al. [1978], the chondrocyte phenotype in culture is quite unstable and degrades with subculture. As a result, it was impossible to better analyze the composition of these structures. We do not exclude the possibility that the multinucleate masses we observed may have been foci of cell types other than cardiac cells. In view of our interest in applying the successful approach of other scientists working with 5-azacytidine and cardiac transformation, our experiments focused only on determining the presence of cardiac-specific troponin and myosin. However, the infrequent and seemingly random appearance of the multinucleate structures in our work attests to the argument by Liu et al. [2003] that genetic reprogramming by 5 -azacytidine may be nonspecific. Because it is a cytosine analogue and appears to work by altering gene methylation [Jones and Taylor, 1980], it is more feasible to hypothesize a sequence of random gene activation than specific cardiac gene potentiation.

The basis for the discrepancy between our results and those of some others is difficult to assess [Constantinides et al., 1977; Constantinides et al., 1978; Taylor and Jones, 1979; Fukuda, 2001; Tomita et al., 2002; Rangappa et al., 2003]. Since we did not carry out a 'control' experiment in an attempt to confirm that, following exposure to 5azacytidine, porcine MSC can differentiate into cardiomyocytes, or compare porcine HSC with their murine counterparts, the negative result we obtained could be associated with technical discrepancies or failures. For example, the particular culture conditions we employed may not have been appropriate for porcine cells. Furthermore, our lack of better 'control' experiments is explained by the considerably low cell yield associated with the purification and separation procedures; it afforded us only enough cells to carry out the 'experimental' segment of our protocol. Increasing the number of experiments would not have addressed this problem, as the issue was one of low cell yield following separation and not necessarily variation between the samples of each tissue examined. A final possibility is that, although we were not able to confirm troponin and myosin expression in these cells at the 5-week time point, other cardiac proteins, such as $\mathrm{Nk} 2.5$ or alpha-actinin, may have been present at other time points during the experiment. As the scope of our study was limited by the commercial availability of porcine markers for these proteins, we were not able to investigate for their presence. As this was an initial study into the 'plasticity' of splenic HSC, we restricted our Western blot analysis to those cardiac proteins described by others and did not attempt detection of proteins that may be expressed by bone, cartilage or endothelial cells. These limitations can only be resolved by further investigations, possibly on a larger scale. 
The goal of our work was not to definitively answer the question of HSC plasticity in a porcine model, but rather to stimulate new thinking with regard to an alternative source of adult stem cells for transdifferentiation studies, namely the adult spleen. One other important difference that may account for variability in results is the donor species. Although Tomita et al. [2002] assessed porcine myocardial function following infarction and the subsequent infusion of 5-azacytidine-treated MSC, the majority of in vitro studies have been in rodent species. Pig BM and splenic cells may not have the same pluripotency or transforming capabilities as those in lower mammals. It is also possible that, in the pig and higher mammals, culture by the method we utilized may drive the cells into a state of senescence, and therefore, are less susceptible to the effects of 5-azacytidine. Additionally, if 5-azacytidine requires a certain state of cellular interaction, enrichment or depletion of c-kit+ stem cells may be detrimental to the intercellular contact or cytokine-mediated microenvironment that may be necessary. The enrichment of c-kit+ cells by MACS may have resulted in the removal or destruction of critical cellular components through specific or nonspecific binding to the column; however, the lack of correlation between fibroblastic growth states and individual c-kit concentrations suggests that other mechanisms may be at work. Finally, only one concentration of 5-azacytidine $(9 \mu M)$ was tested, based upon previous reports that this concentration was the most effective at inducing cardiac phenotype [Rangappa et al., 2003]. As our cells were plated at a higher density than that previously reported [Rangappa et al., 2003], the concentration of 5-azacytidine may not have been sufficient. While the absence of any clear cardiomyocyte phenotype is consistent with the failure to detect either myosin or troponin by Western blot, it is also possible that this technique may not have been sensitive enough to distinguish expression of these proteins by a small subpopulation of cells.

Our study does not preclude the ability of BM and splenic cells to be transformed towards cardiac phenotype. It does, however, suggest that our methodology for the enrichment or depletion of c-kit+ stem cells does not efficiently affect this process.

\section{Conclusions}

Treatment with 5-azacytidine of cultures derived from the porcine BM and spleen led to an unpredictable pattern of growth. Regardless of exposure to 5-azacytidine, fibroblastic activity was present to varying extent among separate populations of c-kit+ and c-kit- populations. Although there were some ball-like formations in both BM and spleen c-kit+ populations that were similar to those observed by others, these cell clusters showed no sign of in vitro beating. Furthermore, neither cardiac troponin nor myosin heavy chain were detected by Western blot in cells derived from either source. These results suggest that, using the protocol we employed, MACS of c-kit+ stem cells and treatment with 5-azacytidine is not an efficient means of inducing cardiac gene expression in porcine HSC derived from either BM or spleen.

\section{Acknowledgments}

The authors thank David H. Sachs, MD, Yong-Guang Yang, $\mathrm{MD} \mathrm{PhD}$, and George Daley, MD PhD, for their critical reviews of the manuscript. M.L.R. received a Milton G. Hendlich Research Fellowship grant from the Office of Enrichment Programs of Harvard Medical School. F.J.M.F.D. was a recipient of grants from the Ter Meulen Fund from the Royal Netherlands Academy of Arts and Sciences, the Prof. Michael van Vloten Fund and the Netherland-America Foundation. D.K.C.C. was a consultant to Immerge BioTherapeutics, Inc. Work in our laboratory was supported in part by NIH Program Project 1PO1 A145897 and by a sponsored research agreement between the Massachusetts General Hospital and Immerge BioTherapeutics, Inc. 


\section{References}

Ashton, B.A., T.D. Allen, C.R. Howlett, C.C. Eaglesom, A. Hattori, M. Owen (1980) Formation of bone and cartilage by marrow stromal cells in diffusion chambers in vivo. Clin Orthop Relat Res 151: 294-307.

Bennett, J.H., C.J. Joyner, J.T. Trifitt, M.E. Owen (1991) Adipocytic cells cultured from marrow have osteogenic potential. J Cell Sci 99: 131139.

Benya, P.D., S.R. Padilla, M.E. Nimni (1978) Independent regulation of collagen types by chondrocytes during the loss of differentiated function in culture. Cell 15: 1313-1321.

Breems, D.A., E.A. Blokland, S. Neben, R.E. Ploemacher (1994) Frequency analysis of human primitive haematopoietic stem cell subsets using a cobblestone area forming cell assay. Leukemia 8: 1095-1104.

Chacko, S., J. Abbott, S. Holtzer, H. Holtzer (1969) The loss of phenotypic traits by differentiated cells. 6. Behavior of the progeny of a single chondrocyte. J Exp Med 130: 417-441.

Constantinides, P.G., P.A. Jones, W. Gevers (1977) Functional striated muscle cells from non-myoblast precursors following 5-azacytidine treatment. Nature 267: 364-366.

-Constantinides, P.G., S.M. Taylor, P.A. Jones (1978) Phenotypic conversion of cultured mouse embryo cells by aza pyrimidine nucleosides. Dev Biol 66: 57-71.

Dor, F.J.M.F., M.L. Ramirez, E.L. Altman (2004) The spleen as a source of hematopoietic stem cells: potential for tolerance induction. Transplantation 78(suppl 1): 590.

Ferrari, G., G. Cuesella-De Angelis, M. Colletta, E. Paolucci, A. Stornaiuolo, G. Cossu, F. Mavilio (1998) Muscle regeneration by bone marrowderived myogenic progenitors. Science 279: $1528-1530$.
Friedenstein, A.J., R.K. Chailakhyan, U.V. Gerasimov (1987) Bone marrow osteogenic stem cells: in vitro cultivation and transplantation in diffusion chambers. Cell Tissue Kinet 20: 263-272.

Fukuda, K. (2001) Development of regenerative cardiomyocytes from mesenchymal stem cells for cardiovascular tissue engineering. Artif Organs 25: 187-193.

Jones, P.A., S.M. Taylor (1980) Cellular differentiation, cytidine analogs and DNA methylation. Cell 20: 85-93.

Kodama, S., W. Kühtreiber, S. Fujimura, E.A. Dale, D.L. Faustman (2003) Islet regeneration during the reversal of autoimmune diabetes in NOD mice. Science 302: 1223-1227.

Le Guern, A.C., M.A. Giovino, M. Abe, P.R. Theodore, J. Qi, J.D. Down, D.H. Sachs, M. Sykes, Y.G. Yang (2003) Stem cell activity of porcine c-kit+ hematopoietic cells. Exp Hematol 31: 833-840.

Leor, J., M. Patterson, M.J. Quinones, L. Kedes, R.A. Kloner (1996) Transplantation of fetal myocardial tissue into the infracted myocardium of a rat. A potential method for repair of infracted myocardium? Circulation 94(suppl II): 332-336.

Liu, Y., J. Song, W. Liu, Y. Wan, X. Chen, C. Hu (2003) Growth and differentiation of rat bone marrow stromal cells: does 5-azacytidine trigger their cardiomyogenic differentiation? Cardiovasc Res 58: 460-468.
Makino, S., K. Fukuda, S. Miyoshi, F. Konishi, H. Kodama, J. Pan, M. Sano, T. Takahashi, S. Hori, H. Abe, J. Hata, A. Umezawa, S. Ogawa (1999) Cardiomyocytes can be generated from stromal cells in vitro. J Clin Invest 103: 697 705 .

Ploemacher, R.E., J.P. van der Slujis, J.S.A. Voerman, N.H. Brons (1989) An in vitro limitingdilution assay for long-term repopulating hematopoietic stem cells in the mouse. Blood 74 . 2755-2763.

Rangappa, S., C. Fen, E.H. Lee, A. Bongso, E.K. Sim (2003) Transformation of adult mesenchymal stem cells isolated from the fatty tissue into cardiomyocytes. Ann Thorac Surg 75: 775-779.

-Rickard, D.J., T.A. Sullivan, B.J. Shenker, P.S. Leboy, I. Kazhdan (1994) Induction of rapid osteoblast differentiation in rat bone marrow stromal cell cultures by dexamethasone and BMP-2. Dev Biol 161: 218-228.

Sachs, D.H. (1992) MHC homozygous miniature swine; in Swindle, M.M., D.C. Moody, L.D. Phillips (eds): Swine as Models in Biomedical Research. Ames, Iowa State University Press, pp 3-15.

Taylor, S.M., P.A. Jones (1979) Multiple new phenotypes induced in 10T1/2 and 3T3 cells treated with 5-azacytidine. Cell 17: 771-779.

Tomita, S., R.K. Li, R.D. Weisel, D.A. Mickle, E.J. Kim, T. Sakai, Z.Q. Jia (1999) Autologous transplantation of bone marrow cells improves damaged heart function. Circulation 100(suppl 19): II247-II256.

Tomita, S., D.A.G. Mickle, R.D. Weisel, Z.Q. Jia, L.C. Tumiati, Y. Allidina, P. Liu, R.K. Li (2002) Improved heart function with myogenesis and angiogenesis after autologous porcine bone marrow stromal cell transplantation. $\mathrm{J}$ Thorac Cardiovasc Surg 123: 1132-1140. 